\title{
Numerical Solution of High-Dimensional Nonlinear Rotor-Bearing Dynamic System with Precise Integration
}

\author{
Guangtian Shi*
}

School of Mechatronic Engineering, Lanzhou Jiaotong University, Lanzhou 730070, P.R. China

\begin{abstract}
Through an example of rotor system which has multi-degree of freedom mounted on the nonlinear fluid film bearings, this paper analyzes the precise integration algorithm, a new numerical solution for high-dimensional nonlinear dynamics system. The precise integration method has advantages of long step, high precision and absolute stability for solving nonlinear dynamics equations. To make good use of the method, firstly, the precise integral iterative formula is given and then the mechanism of controlling high precision and efficiency is discussed. The evolution of precise integration method is an algorithm with explicit, simple form, self-start, and fast to solve nonlinear dynamics equations. High power of athwart of Hamiltonian matrix is not needed, so it is convenient in this case. The stability of period response of nonlinear rotor-bearing system is analyzed by employing the precise integration method. The bifurcation rules of the period response of the elastic rotor system with multi-degree of freedom are obtained and the chaos of the system is determined according to the fractal dimension of Poincare mapping of phase space at a certain speed.
\end{abstract}

Keywords: Chaos, High dimensional nonlinear system, Precise integration, Rotor-bearing system, Stability.

\section{INTRODUCTION}

There are two kinds of classical numerical integration methods, implicit and explicit. The explicit calculation method has character of simple calculation and high efficiency, but it is often at the expense of some accuracy and stability, and the size of time step is limited by the selected stability criteria. The implicit calculation method has the features of good stability and easy selection of time step but every step forward requires solving a large set of linear algebraic equations. Especially, for non-linear problems a recalculation of damping and stiffness matrices and triangular decomposition has to be done. This will cause great difficulties for analysis of a large multi-degree of freedom engineering problems and their computational time and expense is very considerable.

The precise integration method (PIM) discussed in this article is an important explicit method, which is developed in recent years and applied in more and more numerical solutions of dynamics problem. It is used to solve the time step integration for the time-invariant system first. For such a problem, precise integration gives a highly precise numerical result, which approaches the full computer precision [1].

\section{PRECISE INTEGRATION}

Consider the following nonlinear vibration systems

$M \ddot{x}+G \dot{x}+K x=f(t)$

where, $x(0), \dot{x}(0)$ is known, $M, G$ and $K$ are matrices of $n$ and $n, x$ is $n$ dimensional vector, and $f(t)$ is the centrifugal force.

*Address correspondence to this author at the School of Mechatronic Engineering, Lanzhou Jiaotong University, Lanzhou 730070, P.R. China; Tel:+86-931-4938013; Fax:+86-931-4938023; E-mail: shigt@mail.lzjtu.cn
Let

$p=M \dot{x}+G x / 2$.

The equation (1) can be expressed as follows

$\dot{v}=H v+r$

where,

$$
\begin{aligned}
& v=\left\{\begin{array}{l}
x \\
p
\end{array}\right\}, r=\left\{\begin{array}{l}
0 \\
f
\end{array}\right\}, \\
& H=\left\{\begin{array}{cc}
-M^{-1} G / 2 & M^{-1} \\
G M^{-1} G / 4-K & -G M^{-1} / 2
\end{array}\right\} .
\end{aligned}
$$

The precise integration method is suitable for processing a first-order differential equation, so the Hamiltonian system is used commonly for those problems [2,3]. The $v$ in equation (3) is the unknown $2_{n}$ dimensional vector to be solved, and $H$ is called Hamiltonian matrix.

\subsection{Addition Theorem of Matrix Exponential Function}

Addition Theorem

$\exp (H \cdot \tau)=[\exp (H \cdot \tau / n)]^{n}$

Let $n=2^{N}$. When $N=20$, that $\Delta t=\tau / n=9.54 \mathrm{E}-08$ is very small if $\tau=0.1$, so the following equation is obtained for small $\Delta t$.

$\exp (H \cdot \Delta t) \approx I+H \cdot \Delta t+(H \cdot \Delta t)^{2} / 2=I+T_{a}$

where,

$T_{a}=H \cdot \Delta t+(H \cdot \Delta t)^{2} / 2$

It is a very small amount. 
Rewrite the equation (4) as follows for calculation of matrix $T$.

$T=\left[I+T_{a}\right]^{2^{N}}=\left[I+T_{a}\right]^{2^{N-1}} \times\left[I+T_{a}\right]^{2^{N-1}}$

$\left(I+T_{b}\right) \times\left(I+T_{c}\right)=I+T_{b}+T_{c}+T_{b} \times T_{c}$

The matrix $T$ will be obtained by iteration with equation (8) as the following computer algorithm.

$T_{a}=H \cdot \Delta t+(H \cdot \Delta t)^{2} / 2$

for $(i=0 ; i<=N ; i++)$

$T_{a}=2 T_{a}+T_{a} \times T_{a} ; T=I+T_{a} ;$

This algorithm can significantly reduce the accumulated error of computer iterative solver.

\subsection{Precise Integration of Homogeneous Equation}

Known from the theory of ordinary differential equations, the homogeneous equation (9) of equation (3) should be solved firstly.

$\dot{v}=H v$

Rewrite equation (9) as follows:

$\frac{d v}{v}=H d t$

And then the solution of equation (9) is

$v=\exp (H \cdot t) \cdot v_{0}$

If the time step is $\tau$, then

$v(\tau)=\exp (H \cdot \tau) \cdot v_{0}=T \cdot v_{0}$

where,

$T=\exp (H \cdot \tau)$

\subsection{Accuracy Analysis}

The main step of precise integration is the calculation of matrix $T[4,5]$. Therefore the calculation error comes from the expansion equation (5) in addition to error of matrix multiplication. In the calculation of $2 N$ matrix $\mathrm{Ta}$ is calculated with iterative algorithm, and its main item in the beginning is $(H \cdot \Delta t)$, so the calculation error must be compared with it $[6,7]$. Because the next item of expansion equation (5) is $(H \cdot \Delta t)^{3} / 3$ !, the relative calculation error can be roughly estimated to be $(H \cdot \Delta t)^{3} / 3$ ! .

Suppose Eigen solution of matrix $H$ is obtained, then

$$
H=Y[\lambda] Y^{-1}
$$

where, $Y$ is the matrix which is formed by Eigen-vectors and $\lambda$ is the vector of eigenvalues. Then equation (15) is obtained.

$$
\begin{aligned}
\exp (H \cdot \Delta t) & =Y \exp ([\lambda] \cdot \Delta t) Y^{-1} \\
& =Y[\exp (\lambda \cdot \Delta t)] Y^{-1}
\end{aligned}
$$

So the equation (9) corresponds approximately to equation (16). $\exp (\lambda \cdot \Delta t) \approx 1+\lambda \cdot \Delta t+(\lambda \cdot \Delta t)^{2} / 2$

The above calculation error analysis separates the relative error of $(H \cdot \Delta t)^{3} / 3$ ! of every different eigenvalue solution out. Therefore, the value of $[\operatorname{ABS}(\lambda) \cdot \Delta t]^{3}$ of each eigenvalue is very important to the relative calculation error. Because the double precision number is 16 bits in decimal, that $\operatorname{ABS}(\lambda) \cdot \tau / 2^{N}<10^{-4}$ must be satisfied for a double precision number. When $N=20$, that $\operatorname{ABS}(\lambda) \cdot \tau / 2^{N}<10^{-4}$ is satisfied, in which $\lambda$ is $i \omega$ for the un-damped free vibration problem where $\omega$ is the angular frequency. This suggests that even if the integration step is 16 cycles, it does not bring error of expansion equation (9). Of course, a high-frequency vibration $\omega$ should be considered. The fact is that the actual vibration damping exists, so high frequency vibration itself has become insignificant after several cycles of vibration. Therefore, even for high-frequency vibration the error estimate with $(H \cdot \Delta t)^{3} / 3$ ! is too conservative. That is, the accuracy of precise integration method is very high.

\subsection{Precise Integration for Nonlinear Dynamic Equation}

Reference [8] established a nonlinear dynamic system, and then used an improved precise integration method to solve the nonlinear dynamic system. The nonlinear dynamic system was converted into an augmented Lie type dynamic system and then the precise integration method is improved for solving the above augmented equation and preserving its group structure in the meantime.

The final numerical solution formula for equation (2) can be drawn with precise integration method [9] as follows:

$$
v_{(k+1)}=e^{H \tau}\left[v_{(k)}+\sum_{j=0}^{\infty}\left(\sum_{m=0}^{\infty}(-1)^{m} \frac{\tau^{m+j+1}}{j ! m !(m+j+1)} H^{m}\right) f_{(k)}^{(j)}\right]
$$

The above iterative formula has matrix $\mathrm{H}$ with higher power, so a further derived form for the solution vector $v$ at time $t$ is obtained as follows [2].

$$
\begin{aligned}
& v(t)=e^{H \tau}\left(v_{(k)}+\sum_{j=0}^{\infty}\left(H^{-1}\right)^{j+1} f_{(k)}^{(j)}\right)- \\
& \sum_{j=0}^{\infty}\left(\sum_{m=1}^{j+1} \frac{\tau^{j+1-m}}{(j+1-m) !} H^{-m}\right) f_{(k)}^{(j)} \\
& t_{k} \leq t \leq t+\Delta t, 0 \leq \tau \leq \Delta t
\end{aligned}
$$

In practical calculation the series only take limited items. For convenience, when the number $j$ of Taylor polynomials is 1 and 2 and so on, the corresponding solution formula is called the first approximation formula and the second approximation formula and so on as follows:

$$
v=e^{H \tau}\left(v_{(k)}+H^{-1} f_{(k)}\right)-H^{-1} f_{(k)}
$$




$$
\begin{aligned}
& v=e^{H \tau}\left(v_{(k)}+H^{-1} f_{(k)}+H^{-2} f_{(k)}\right)- \\
& \left(H^{-1} f_{(k)}+\left(\tau H^{-1}+H^{-2}\right) f_{(k)}\right)
\end{aligned}
$$

Formula (19) uses $v_{(k)}$ and $f_{(k)}$ which have been obtained, so it is a convenient self-starting formula. When $f$ is a constant, the first approximation formula is equivalent to a numeric expression of exact solution, i.e., the accuracy of the first approximation formula (19) is unrelated to the size of time step, $\tau$.

Precise integration method is unconditionally stable in numerical methods.

\subsection{Dynamic Characteristic Analysis of a Rotor-Bearing System}

Nonlinear dynamics and stability of the rotor-bearing system, which is simplified as the Jeffcott rotor, are investigated both theoretically and experimentally. The nonlinear oil-film forces are obtained under the short bearing theory. The precise integration is utilized to solve the nonlinear governing equations. Bifurcation diagrams, waterfall plots, Poincare maps, spectrum plots and rotor orbits are drawn to analyze various nonlinear phenomena and system unstable processes [10].

In this paper, Capone nonlinear oil film force model of cylindrical bearing is given [11]. In 1991 Capone made Nonlinear Oil Film Force Modeled under the assumption of short bearing, as shown in Fig. (1). The model has better accuracy and convergence, and some scholars have proved the reliability of the model [12].

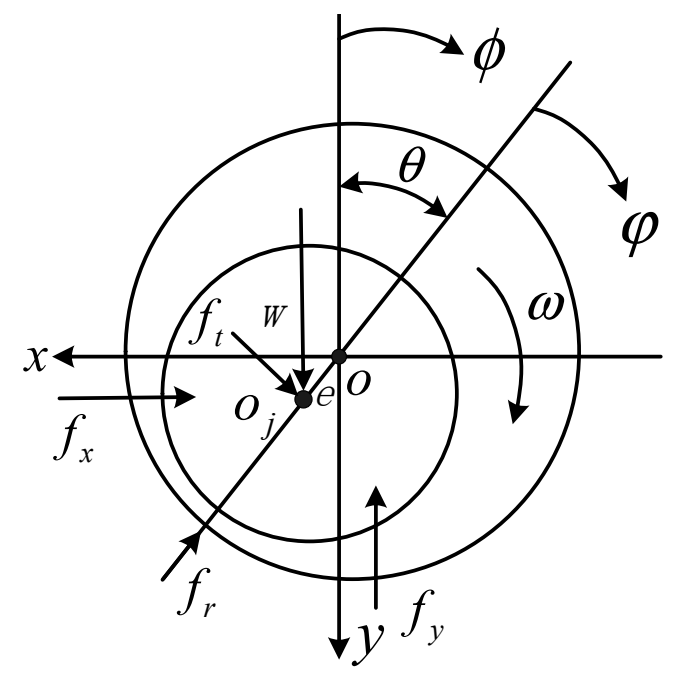

Fig. (1). Calculation coordinates of bearing.

Dimensionless Reynolds equation under the assumption of short bearing is as follows:

$\left(\frac{R}{L}\right)^{2} \frac{\partial}{\partial z}\left(h^{3} \frac{\partial p}{\partial z}\right)=$

$x \sin \vartheta-y \cos \vartheta-2\left(x^{\prime} \cos \vartheta+y \sin \vartheta\right)$

The press force from equation (21) is as follows:
$p=\frac{1}{2}\left(\frac{L}{D}\right)^{2} \frac{\left(x-2 y^{\prime}\right) \sin \vartheta-\left(y+2 x^{\prime}\right) \cos \vartheta}{(1-x \cos \vartheta-y \sin \vartheta)^{3}}\left(4 z^{2}-1\right)$

The formula of dimensionless nonlinear oil film force is as follows:

$$
\begin{aligned}
& \left\{\begin{array}{l}
f_{x} \\
f_{y}
\end{array}\right\}=-\frac{\left[\left(x-2 y^{\prime}\right)^{2}+\left(y+2 x^{\prime}\right)^{2}\right]^{1 / 2}}{1-x^{2}-y^{2}} \bullet \\
& \left\{\begin{array}{l}
3 x V(x, y, \alpha)-\sin \alpha G(x, y, \alpha)-2 \cos \alpha S(x, y, \alpha) \\
3 y V(x, y, \alpha)+\cos \alpha G(x, y, \alpha)-2 \sin \alpha S(x, y, \alpha)
\end{array}\right\}
\end{aligned}
$$

where,

$$
\begin{aligned}
& V(x, y, \alpha)=\frac{2+(y \cos \alpha-x \sin \alpha) G(x, y, \alpha)}{1-x^{2}-y^{2}}, \\
& S(x, y, \alpha)=\frac{x \cos \alpha+y \sin \alpha}{1-(x \cos \alpha+y \sin \alpha)^{2}}, \\
& G(x, y, \alpha)=\frac{2}{\left(1-x^{2}-y^{2}\right)^{1 / 2}}\left[\frac{\pi}{2}+\operatorname{arctg} \frac{y \cos \alpha-x \sin \alpha}{\left(1-x^{2}-y^{2}\right)^{1 / 2}}\right], \\
& \alpha=\operatorname{arctg} \frac{y+2 x^{\prime}}{x-2 y^{\prime}}-\frac{\pi}{2} \operatorname{sign}\left(\frac{y+2 x^{\prime}}{x-2 y^{\prime}}\right)-\frac{\pi}{2} \operatorname{sign}\left(y+2 x^{\prime}\right)
\end{aligned}
$$

Fig. (2) shows a mathematical model of nonlinear rotorbearing system [13], where $m_{1}$ is mass quality focused on the rotor bearings, $c_{1}$ is rotor structural damping at the bearings, $k_{p}$ is the shaft stiffness, $f_{x}, f_{y}$ are nonlinear oil film force components, $m_{2}$ is mass quality of rotor disc, $c_{2}$ is damping of rotor disc. When the rotor angular velocity is $\omega$ and dimensionless eccentricity, $\rho_{1}=e_{1} / c, \rho_{2}=e_{2} / c$.

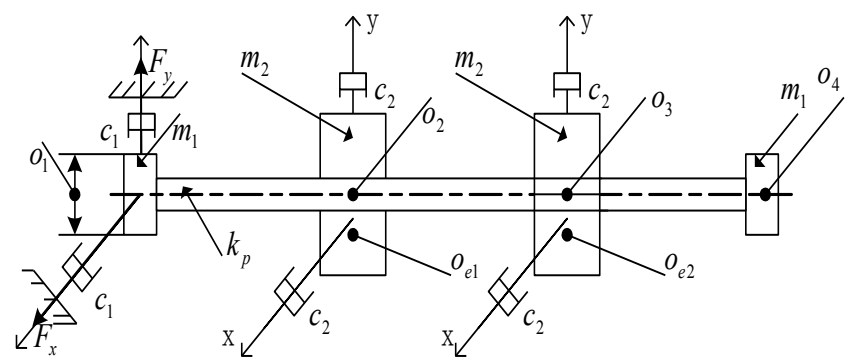

Fig. (2). Model of an elastic rotor-bearing system.

The system dimensionless equation under the assumption of symmetry is as follows:

$M \ddot{x}+C \dot{x}+K x=F+Q$

where, $M, C$ and $K$ are respectively mass, damping and stiffness matrices of 8 and 8 dimensions, and $x$ is displacement matrix of 8 and 1 dimensions, and $F$ is nonlinear oil film force matrix of 8 and 1 dimensions, $Q$ is force matrix of 8 and 1 dimensions changing with time which may be an unbalanced force or other incentives. 
$M=\left[\begin{array}{llllllll}1 & 0 & 0 & 0 & 0 & 0 & 0 & 0 \\ 0 & 1 & 0 & 0 & 0 & 0 & 0 & 0 \\ 0 & 0 & 1 & 0 & 0 & 0 & 0 & 0 \\ 0 & 0 & 0 & 1 & 0 & 0 & 0 & 0 \\ 0 & 0 & 0 & 0 & 1 & 0 & 0 & 0 \\ 0 & 0 & 0 & 0 & 0 & 1 & 0 & 0 \\ 0 & 0 & 0 & 0 & 0 & 0 & 1 & 0 \\ 0 & 0 & 0 & 0 & 0 & 0 & 0 & 1\end{array}\right]$,

$C=\left[\begin{array}{cccccccc}a_{1} / m_{1} & 0 & 0 & 0 & 0 & 0 & 0 & 0 \\ 0 & a_{1} / m_{1} & 0 & 0 & 0 & 0 & 0 & 0 \\ 0 & 0 & a_{3} / m_{2} & 0 & 0 & 0 & 0 & 0 \\ 0 & 0 & 0 & a_{3} / m_{2} & 0 & 0 & 0 & 0 \\ 0 & 0 & 0 & 0 & a_{3} / m_{2} & 0 & 0 & 0 \\ 0 & 0 & 0 & 0 & 0 & a_{3} / m_{2} & 0 & 0 \\ 0 & 0 & 0 & 0 & 0 & 0 & a_{1} / m_{1} & 0 \\ 0 & 0 & 0 & 0 & 0 & 0 & 0 & a_{1} / m_{1}\end{array}\right]$

$K=\left[\begin{array}{cccccccc}a_{2} / m_{1} & 0 & -a_{2} / m_{1} & 0 & 0 & 0 & 0 & 0 \\ 0 & a_{2} / m_{1} & 0 & -a_{2} / m_{1} & 0 & 0 & 0 & 0 \\ -a_{2} / m_{2} & 0 & 2 a_{2} / m_{2} & 0 & -a_{2} / m_{2} & 0 & 0 & 0 \\ 0 & -a_{2} / m_{2} & 0 & 2 a_{2} / m_{2} & 0 & -a_{2} / m_{2} & 0 & 0 \\ 0 & 0 & -a_{2} / m_{2} & 0 & 2 a_{2} / m_{2} & 0 & -a_{2} / m_{2} & 0 \\ 0 & 0 & 0 & -a_{2} / m_{2} & 0 & 2 a_{2} / m_{2} & 0 & -a_{2} / m_{2} \\ 0 & 0 & 0 & 0 & -a_{2} / m_{1} & 0 & a_{2} / m_{1} & 0 \\ 0 & 0 & 0 & 0 & 0 & -a_{2} / m_{1} & 0 & a_{2} / m_{1}\end{array}\right]$

$x=\left[\begin{array}{l}x_{1} \\ y_{1} \\ x_{2} \\ y_{2} \\ x_{3} \\ y_{3} \\ x_{4} \\ y_{4}\end{array}\right]$

$F=\frac{1}{m_{11}}\left[\begin{array}{llllllll}f_{x 1} & f_{y 1}-G & 0 & 0 & 0 & 0 & f_{x 4} & f_{y 4}-G\end{array}\right]^{T}$

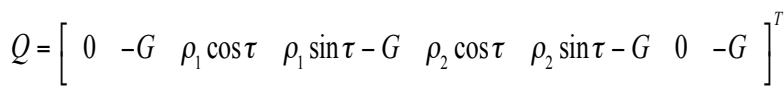

where, $x_{i}=\frac{X_{i}}{c}$ and $y_{i}=\frac{Y_{i}}{c}$ are dimensionless displacements, $f_{x}=\frac{F_{x}}{\delta}, f_{y}=\frac{F_{y}}{\delta}$ are dimensionless nonlinear oil film force components, $\mu$ is lubricant viscosity, $G=\frac{g}{c \omega^{2}} \quad$ is dimensionless force, $\tau=\omega t$ is dimensionless time, $e_{1}, e_{2}$ are amount of eccentricity, $\mathrm{c}$ is bearing radial clearance, $\mathrm{L}$ is bearing length, $\mathrm{R}$ is bearing radius, $\delta=\frac{\mu \omega R L}{m_{1} g}\left(\frac{R}{c}\right)^{2}\left(\frac{L}{2 R}\right)^{2}$ is Sommerfeld amendment number, and $m_{11}=\frac{\omega^{2} c}{\delta g}, a_{1}=\frac{c_{1}}{\omega}$, $a_{2}=\frac{k_{p}}{\omega^{2}}, a_{3}=\frac{c_{2}}{\omega}$, and $f_{x i}, f_{y i}(i=1,4)$ are determined by equation (23).
Let $\quad m_{1}=50 \mathrm{~kg}, \quad m_{1}=210 \mathrm{~kg}, \quad D=114 \mathrm{~mm}, \quad L=56.5 \mathrm{~mm}$, $c=0.2 \mathrm{~mm}, \quad \mu=0.018 \mathrm{~Pa} \cdot \mathrm{s}, \quad k_{p}=165200 \mathrm{kN} / \mathrm{m}, \quad c_{l}=4.3 \mathrm{kN} \cdot \mathrm{s} / \mathrm{m}$, $c_{2}=9.3 \mathrm{kN} \cdot \mathrm{s} / \mathrm{m}, \quad e_{1}=0.06 \mathrm{~mm}$ and $e_{2}=0.08 \mathrm{~mm}$. Precise integration method is used to analyze the response of this rotor-bearing system.

Fig. (3) shows the bearing center displacement of Poincare map when the rotational speed is $400 \mathrm{rad} / \mathrm{s}$, and it can be seen that the system has the characteristics of chaos.
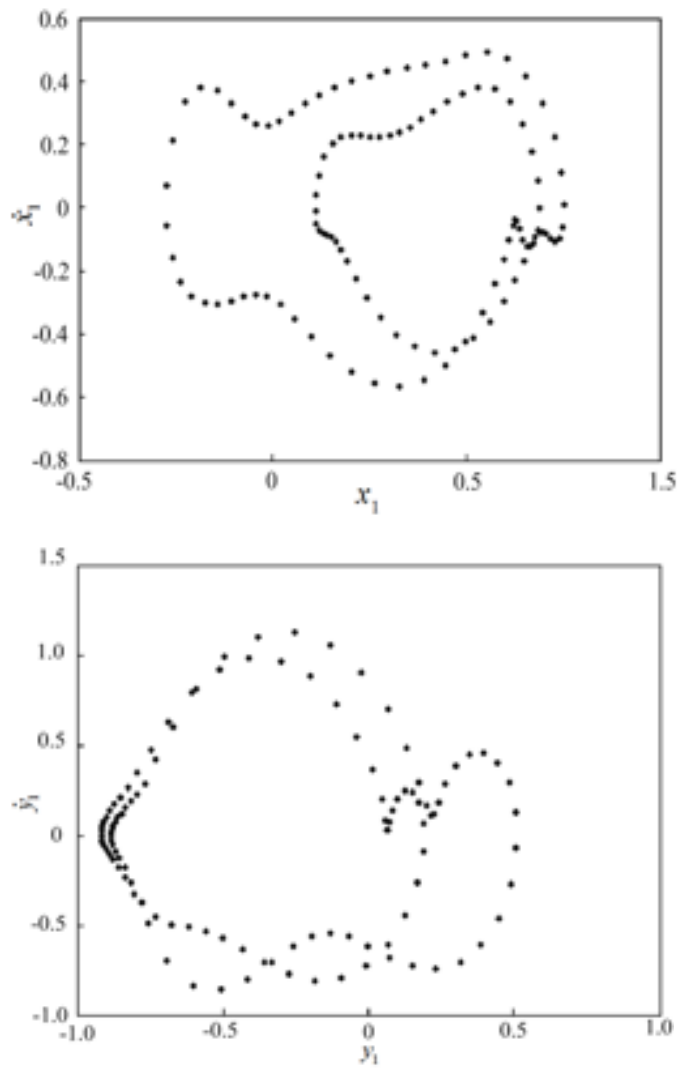

Fig. (3). Poincare map of bearing center for rotational speed of $400 \mathrm{rad} / \mathrm{s}$.

Fig. (4) shows the orbit of bearing and disk center when the rotational speed is $680 \mathrm{rad} / \mathrm{s}$, and it can be seen that the axis movement of rotor and bearing is periodic motion under current rotational speed.

Fig. (5) shows the bearing center displacement of Poincare map when the rotational speed is $845 \mathrm{rad} / \mathrm{s}$, and it can be seen that they present discrete points within a certain range and indicate that the bearing center produces a chaotic motion in the current rotational speed.

Fig. (6) shows the bearing center displacement of Poincare map when the rotational speed is $1040 \mathrm{rad} / \mathrm{s}$, and it can be seen that they present torus and indicate that the bearing center produces a quasi-periodic motion or almost periodic motion in the current rotational speed.

As it can be seen from the above results, the movement of the rotor-bearing system is a complex dynamic behavior that shows bifurcation, chaos and other nonlinear dynamical phenomena due to the rotor unbalance of eccentric mass and bearing oil film force of nonlinear effects, which may lead to system instability [14]. 

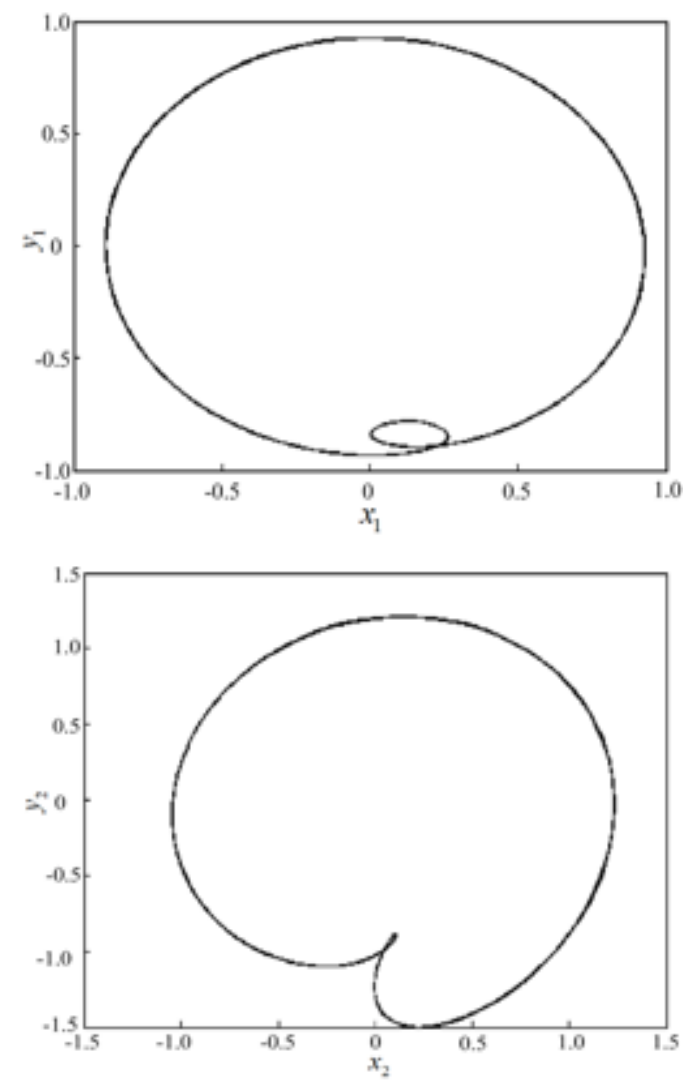

Fig. (4). Orbit of bearing and disk center for rotational speed of $680 \mathrm{rad} / \mathrm{s}$.
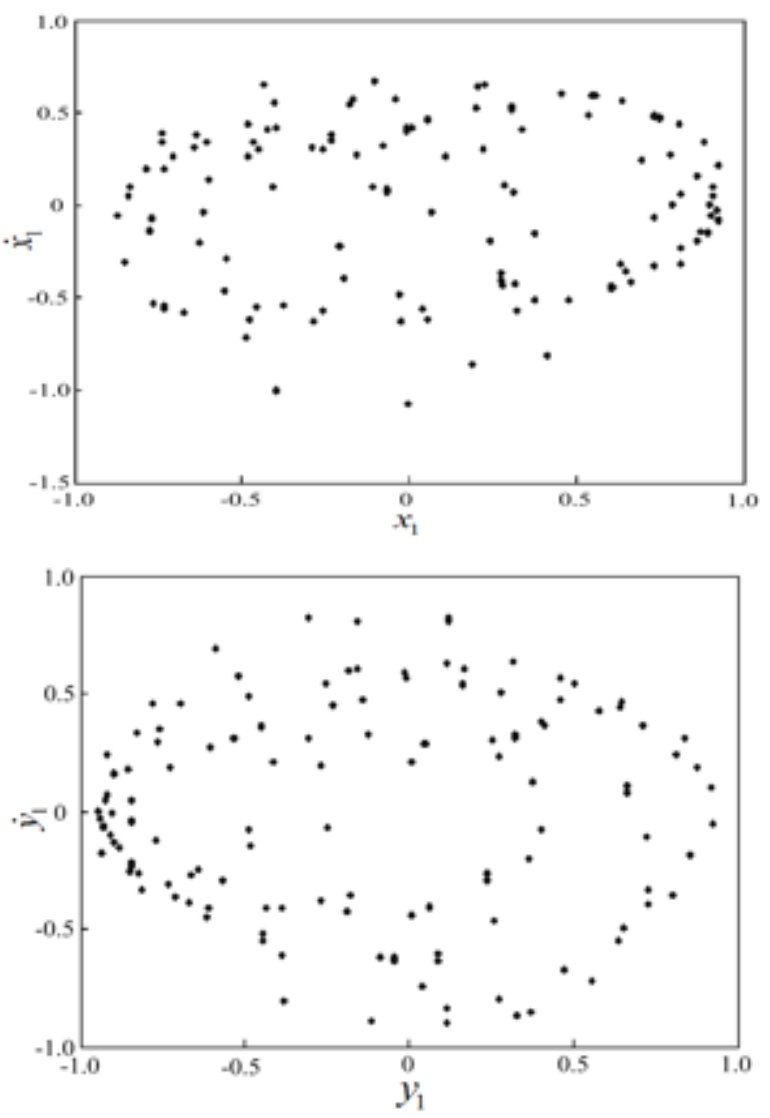

Fig. (5). Poincare map of bearing center for rotational speed of $845 \mathrm{rad} / \mathrm{s}$.
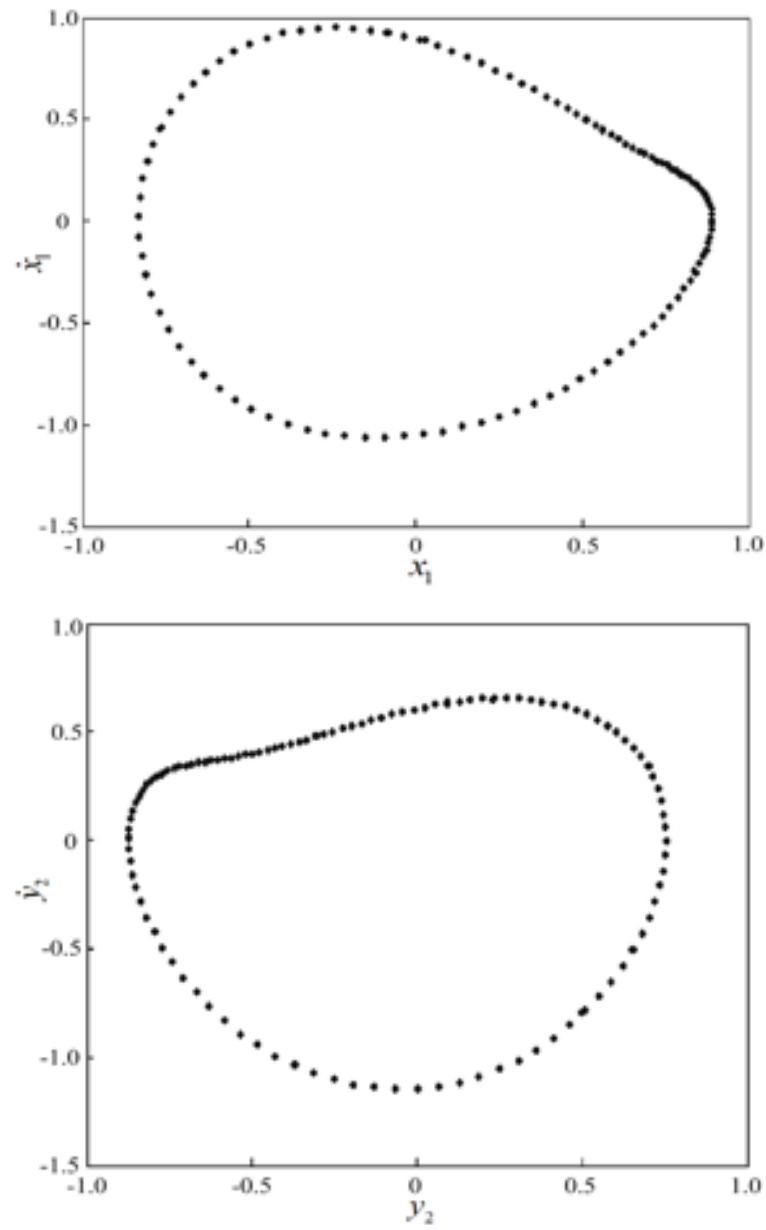

Fig. (6). Poincare map of bearing center for rotational speed of $1040 \mathrm{rad} / \mathrm{s}$.

\section{CONCLUSION}

Precise integration method is proposed by academician Wanxie Zhong in recent years and it has higher accuracy within numerical integration methods. In order to better apply the precise integration to solve nonlinear dynamics problem, this paper analyzes the accuracy and stability of the precise integration method. It explores the internal mechanism of the algorithm and how to achieve its fine precision and high efficiency. Through an example of continuous rotor-bearing system it is proved that precise integration method can be used with a larger time step and it has the advantages of absolute stability of calculation. For differential equations with constant coefficients the state at any time can be obtained by iteration with high computational accuracy, efficiency and numerical stability. Numerical results for the example show that when the rotorbearing system is running, the movement of the rotorbearing axis has a complex dynamic behavior that sequentially shows periodic response, doubling bifurcation and chaotic motions and other dynamic phenomena because of the role of imbalance eccentric mass quality and nonlinear oil film force and other factors with the change of rotation angular velocity. The kinetic behavior of the system with speed changes may lead to system instability. Therefore, the design of the rotor system must make the working speed to avoid these changes in the dynamic behavior of the system. 


\section{CONFLICT OF INTEREST}

The authors confirm that this article content has no conflict of interest.

\section{ACKNOWLEDGEMENTS}

Declared none.

\section{REFERENCES}

[1] W.X. Zhong, "On precise integration method", J. Comput. Appl. Math., vol. 163, no. 1, pp. 59-78, 2004.

[2] H.X. Lv, Z.Q. Cai, and C.H. Qiu, "An explicit precise integration algorithm for Nonlinear Dynamics Problems", Chin. J. Appl. Mech., vol. 18, no. 2, pp. 34-40, 2001.

[3] H.X. Lv, H.J. Yu, and C. H. Qiu, "Solving method and integral equations of nonlinear dynamics of precise integration", Chin. J. Solid Mech., vol. 22, no. 3, pp. 303-308, 2001.

[4] Y. Xiang, Y.Y. Huang, and G.W. Zeng, "Error analysis and precision design in precise integration method", Chin. J. Comput. Mech., vol. 19, no. 3, pp. 276-230, 2002.

[5] M. Wen, and Z.C. Deng, "Application of improved precise integration method in the mechanical vibration", Mech. Sci. Technol., vol. 21, no. 1, pp. 40-41, 2002.
[6] H.W. Zhang, "Discussion on accuracy of dynamic analysis with precise integration algorithm", J. Mech., vol. 33 , no. 6, pp. 848852, 2001.

[7] Z.Q. Cai, Y.X. Gu, and W.X. Zhong, "Precise integration method for a class of nonlinear periodic system response", Mech., vol. 21, no. 2, pp.20-23, 2000.

[8] S.Y. Zhang, and Z.C. Deng, "An improved precise integration method for nonlinear dynamic system", Mech. Res. Commun., vol. 30, no. 1, pp. 33-38, 2003.

[9] G.T. Shi, "Research on nonlinear dynamics of machine tool", $\mathrm{PhD}$ thesis, Lanzhou University, 2002.

[10] X.Y. Shen, J.H. Jia, M. Zhao, and J.P. Jing, "Experimental and numerical analysis of non- linear dynamics of rotor-bearingseal", Nonlinear Dynam., vol. 53, no. 1-2, pp. 31-44, 2008.

[11] G. Capone "Descrizion Analitica Del Campo Di Forze Fluidodinamico Nei Cuscinetti Cilindrici Lubrificati", L'Energia Elettrica. vol. 3, pp. 105-110, 1991.

[12] G. Adiletta and A. R. Guido, "Chaotic motions of a rigid rotor in short journal bearings", Journal of Nonlinear Dynamics, vol. 10, pp. 251-269, 1996.

[13] X.J. Zhang, "Research on nonlinear dynamics of rotor-bearing system", PhD thesis, Haerbin Institute of Technology, 2001.

[14] L.G. Wang, "Analysis of nonlinear dynamics of rotor-bearing system", PhD thesis, Haerbin Institute of Technology, 2005.

(c) Guangtian Shi; Licensee Bentham Open.

This is an open access article licensed under the terms of the Creative Commons Attribution Non-Commercial License (http://creativecommons.org/licenses/by-nc/4.0/) which permits unrestricted, non-commercial use, distribution and reproduction in any medium, provided the work is properly cited. 